Stoa

Vol. 12, no. 23, 2021, pp. 36-50

ISSN 2007-1868

\title{
ALGUNOS APUNTES SOBRE LA ELECCIÓN DE SISTEMAS LÓGICOS
}

\author{
Some Remarks on the Choice of Logical Systems
}

José Martín CASTRo MANZANo

Universidad Popular Autónoma del Estado de Puebla

jmcmanzano@hotmail.com

RESUMEN: Asumiendo el planteamiento general del pluralismo lógico, en esta contribución presentamos los resultados de una serie de entrevistas a un conjunto de lógicas y lógicos de México. Las entrevistas han sido diseñadas ex professo para aplicar la técnica del Proceso Analítico Jerárquico y con ello obtener modelos de decisión para observar las razones detrás de la elección de un sistema lógico. Como consecuencia de estos modelos presentamos algunas ideas sobre una noción de pluralismo lógico.

PALABRAS CLAVE: Pluralismo lógico · proceso analítico jerárquico · lógica clásica lógica no-clásica $\cdot$ toma de decisiones.

ABSTRACT: By assuming the general outset of logical pluralism, in this contribution we present a series of interviews applied to a set of Mexican logicians. The interviews have been specially designed to make use of the Analytic Hierarchy Process as to obtain decision models about the choice of a logical system. As a consequence we present some ideas about the notion pluralism in logic.

KEYWORDS: Logical pluralism · analytic hierarchy process · classical logic $\cdot$ nonclassical logic $\cdot$ decision-making.

\section{Introducción}

Aunque la lógica clásica cuenta con una tradición que la ha situado en un lugar privilegiado (cf. Eklund, 1996), el surgimiento de diferentes sistemas ha puesto en duda su hegemonía. Ante esta situación, el pluralismo lógico ha 
planteado, grosso modo, que hay más de una lógica (Beall y Restall, 2000; 2006; Goddu, 2002; Field, 2009).

Este planteamiento es complejo y dista de ser trivial, pero aunque se ha centrado en los argumentos para elegir un sistema sobre otro, poco se ha fijado en el proceso de toma de decisiones. Dada esta situación, en este trabajo revisamos una cara social del pluralismo lógico mediante la presentación de los resultados de una serie de entrevistas aplicadas a un conjunto selecto de cuatro lógicas y lógicos de México. La motivación detrás de estas entrevistas es fundamentalmente valorativa: en un horizonte de pluralidad, ¿qué favorecen las y los lógicos cuando hacen lógica?

Con la finalidad de guiar esta motivación las entrevistas fueron diseñadas ex professo para aplicar la técnica del Proceso Analítico Jerárquico y con ello obtener modelos de decisión para observar las razones detrás de la elección de un sistema sobre otro. Adicionalmente, como consecuencia de estos modelos presentamos algunas ideas sobre una noción de pluralismo lógico.

Para alcanzar nuestra meta hemos organizado el trabajo del siguiente modo. Primero hacemos una breve presentación de la técnica que empleamos y de la estructura de la entrevista, posteriormente exponemos los modelos de cada entrevista, y por último, concluimos con una interpretación de los mismos.

\section{El Proceso Analítico Jerárquico}

El Proceso Analítico Jerárquico (PAJ) es un método para la toma de decisiones que produce como salida un modelo de elección jerárquico mediante la identificación de un objetivo, un conjunto de criterios y un conjunto de alternativas (Satty, 2008). Posee un sólido sustento matemático, ofrece la posibilidad de medir criterios cuantitativos y cualitativos y es sumamente fácil de implementar.

Como otros sistemas de decisión multicriterio, no nos garantiza encontrar una elección óptima, pues sus resultados se definen en función de las preferencias (representadas mediante una escala de valores como en la tabla 1) de las y los agentes que eligen; pero sí nos permite medir la consistencia de tales preferencias. 


\begin{tabular}{cc}
\hline Valor & Interpretación \\
\hline 1 & Los elementos son de igual importancia \\
\hline 3 & Un elemento tiene moderada importancia sobre otro \\
\hline 5 & Un elemento tiene alta importancia sobre otro \\
\hline 7 & Un elemento tiene muy alta importancia sobre otro \\
\hline 9 & Un elemento tiene extrema importancia sobre otro \\
\hline $2,4,6,8$ & Valores intermedios \\
\hline Incrementos de 0.1 & Valores intermedios para valoracion más fina \\
\hline & Tabla 1: Escala de Saaty
\end{tabular}

A modo de ejemplo, supongamos que una agente tiene como objetivo comprar un vehículo nuevo, que sus alternativas son los autos A, B, C y D; y que sus criterios son el costo, el diseño y la seguridad. De acuerdo con la técnica del PAJ, la agente debe comparar sus criterios con base en sus preferencias. Así, debe comenzar preguntando qué tanto prefiere el costo sobre el diseño, el costo sobre la seguridad y el diseño sobre la seguridad. Posteriormente, ha de comparar las alternativas con respecto a cada criterio. Así pues, en seguida tiene que comparar, digamos, las alternativas A y B con respecto al diseño, al costo y la seguridad; posteriormente, las alternativas A y $\mathrm{C}$ con respecto a los mismos criterios; y así sucesivamente, hasta agotar todas las combinaciones posibles.

Para seguir con este ejemplo, supongamos que después de aplicar el PAJ obtenemos los resultados de la tabla 2. Estos resultados inducen un modelo de decisión en el que el criterio más importante a considerar cuando se busca comprar un auto resulta ser el diseño (después viene el costo y por último la seguridad) y que la alternativa a elegir es D (y después, A, B y C). Adicionalmente, como la proporción de consistencia (CR por consistency ratio) es mayor que .1000 decimos que, aunque tenemos un modelo, no es consistente, lo cual, a su vez, indica que la agente debería revisar algunas de sus elecciones.

\begin{tabular}{cccc}
\hline Criterios & Índice & Alternativas & Índice \\
\hline Diseño & .5317 & $\mathrm{D}$ & .3305 \\
\hline Costo & .3220 & $\mathrm{~A}$ & .2817 \\
\hline Seguridad & .1463 & $\mathrm{~B}$ & .2001 \\
\hline
\end{tabular}




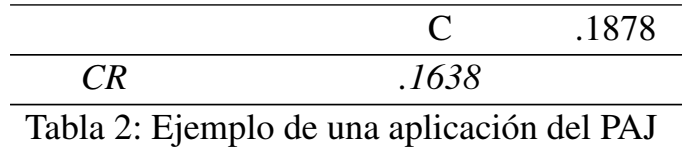

\section{Alternativas, criterios e instrumento}

Las alternativas que nos interesan en este trabajo son sistemas lógicos. Los sistemas lógicos son herramientas formales que nos permiten modelar y estudiar la relación de inferencia. Ejemplos de sistemas lógicos en un entorno plural son, verbigracia, la lógica clásica, la lógica intuicionista y la lógica relevante.

Ciertamente, puesto que las variaciones y diferencias entre sistemas son más ricas e interesantes de lo que podríamos exponer aquí, esta caracterización es bastante superficial; sin embargo, su generalidad nos basta para atisbar un horizonte de diversidad que podemos ilustrar con tres esquemas: la ya tradicional lista de Haack (1978, p. 4) (figura 1a), el retículo de sistemas modales normales de Hughes y Cresswell (1996, p. 367) (figura 1b) o la jerarquía de Moss (2015) para sistemas de primer orden (figura 1c). Por supuesto, como se verá en la siguiente sección, estas ilustraciones ni siquiera bastan para exponer la variedad de alternativas.

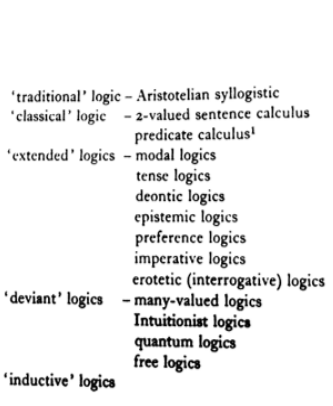

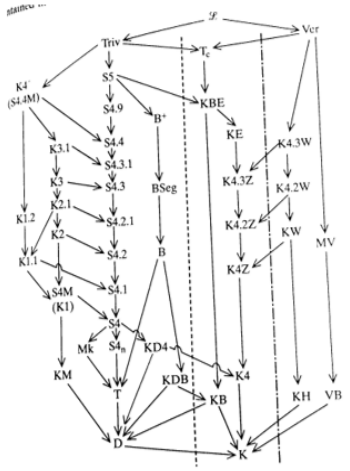

b

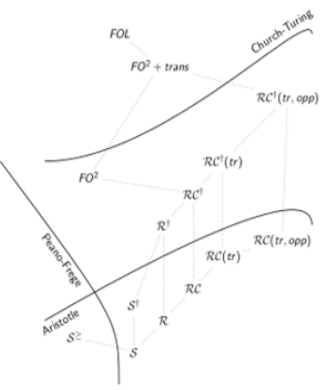

c

Figura 1. Esquemas de alternativas 
Los criterios que nos importan en este estudio pueden definirse en términos de las propiedades metalógicas que se predican de un sistema lógico. Por ejemplo, si tomamos a la lógica proposicional clásica notamos que cuenta con las propiedades metalógicas de consistencia (i.e. que en el sistema no se puede demostrar una proposición y su contradicción), corrección (i.e. que todo teorema es una verdad lógica), completud (i.e. que toda verdad lógica es un teorema) y decidibilidad (i.e. que existe un algoritmo efectivo para determinar si una proposición es una verdad lógica).

La observancia de estas propiedades es relevante porque resulta útil para comparar diferentes sistemas. Así, por ejemplo, observando estas características podemos notar ciertas relaciones entre la lógica proposicional clásica (PROP), la lógica de primer orden (FOL) y la lógica de segundo orden (SOL): FOL tiene menos propiedades que PROP y, a su vez, SOL tiene menos propiedades que FOL (figura 2a).

Sin embargo, no todo se decide en términos de estas propiedades. Lo que ocurre en la práctica de la lógica es más complejo: parece que se asume un canje de atributos en el que se pierden ciertas propiedades pero se ganan otras, como aplicabilidad y expresividad, por ejemplo (figura 2b). Así, PROP es una alternativa consistente (Con), correcta (Cor), completa (Com) y decidible (Dec), pero muy poco expresiva (Exp); mientras que en el otro extremo tenemos a SOL, con un nivel de expresividad muy alto, pero con menos propiedades metalógicas deseables.

Por supuesto, si añadiéramos más sistemas al espacio de decisiones la situación se haría más complicada, pero el problema seguiría siendo similar en el sentido de que siempre existe una situación de compensación o de canje de propiedades que no es trivial (figura $2 \mathrm{c}$ ).

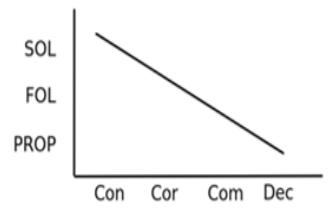

a

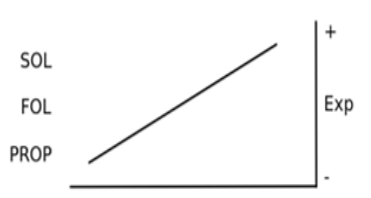

b

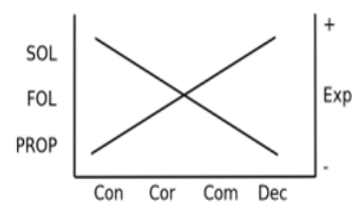

C

Figura 2. Esquemas de criterios

Pues bien, asumiendo esta descripción general del PAJ, de las alternativas y de los criterios, a continuación exponemos la estructura general del instru- 
mento que usamos como entrevista y algunas de las características de las y los participantes del estudio.

La entrevista fue diseñada ex professo para aplicar la técnica del PAJ, por lo que cada pregunta tenía como propósito obtener alguno de los elementos que forman parte estructural de la técnica empleada (tabla 3 ).

\begin{tabular}{cl}
\hline \multicolumn{1}{c}{ Pregunta } & \multicolumn{1}{c}{ Propósito } \\
\hline ¿Qué sistemas lógicos toma en cuenta en su \\
práctica? & Obtener alternativas \\
\hline $\begin{array}{c}\text { ¿Qué propiedades toma en cuenta al momento } \\
\text { de elegir un sistema lógico? }\end{array}$ & Obtener criterios \\
\hline $\begin{array}{c}\text { ¿Cuál es la importancia que le asigna a cada } \\
\text { propiedad con respecto otra? }\end{array}$ & Comparar criterios \\
\hline $\begin{array}{c}\text { ¿Cuál es la importancia que le asigna a cada } \\
\text { alternativa con respecto a cada criterio? }\end{array}$ & $\begin{array}{l}\text { Comparar alternativas } \\
\text { c.r.a criterios }\end{array}$ \\
\hline
\end{tabular}

Tabla 3: Estructura general del instrumento

Cada entrevista fue aplicada in situ, de manera individual e independiente. Al momento de la aplicación los sujetos participaban como docentesinvestigadores en centros universitarios y la mayoría de ellos pertenecía al Sistema Nacional de Investigadores (SNI) del Consejo Nacional de Ciencia y Tecnología del país (CONACyT). Dos realizaban investigación en el Área IV y dos en el Área VII, como se definen en el CONACyT (tabla 4).

\section{Modelos y resultados}

A continuación exponemos los modelos obtenidos, pero antes es necesario aclarar que el instrumento esquematiza únicamente un modelo jerárquico y que de ninguna manera define la respuesta última y definitiva de las y los entrevistados.

Modelo 1. El sujeto 1 consideró seis sistemas y seis propiedades. Los sistemas que tomó en cuenta son los siguientes: la lógica tetravalente, la lógica clásica (FOL), la lógica algebraica, la lógica intensional, la lógica intuicionista y la teoría de conjuntos; y consideró, entre las propiedades, a la expresividad, la explicatividad, la aprioricidad, la objetividad, la simetría y la analiticidad.

Por expresividad entendía a la capacidad que tiene un sistema para representar información. Por explicatividad, la capacidad que tiene un sistema para 


\begin{tabular}{|c|c|c|c|c|c|}
\hline Sujeto & Género & Institución & Área & SNI & Investigación \\
\hline 1 & M & $\begin{array}{c}\text { Nivel } \\
\text { superior-Pública }\end{array}$ & $\begin{array}{l}\text { IV. Humanidades y } \\
\text { Ciencias de la } \\
\text { Conducta (Filosofía) }\end{array}$ & 1 & $\begin{array}{c}\text { Filosofía de las } \\
\text { matemáticas, filosofía de } \\
\text { la lógica, lógica aplicada y } \\
\text { filosofía del lenguaje }\end{array}$ \\
\hline 2 & M & $\begin{array}{c}\text { Nivel } \\
\text { superior-Privada }\end{array}$ & $\begin{array}{l}\text { VII. Ingenierías } \\
\text { (Ciencias } \\
\text { computacionales) }\end{array}$ & 2 & $\begin{array}{l}\text { Representación del } \\
\text { conocimiento, } \\
\text { programación lógica, } \\
\text { razonamiento automático } \\
\text { y argumentación }\end{array}$ \\
\hline 3 & $\mathrm{~F}$ & $\begin{array}{c}\text { Nivel } \\
\text { superior-Pública }\end{array}$ & $\begin{array}{l}\text { VII. Ingenierías } \\
\text { (Ciencias } \\
\text { computacionales) }\end{array}$ & - & $\begin{array}{c}\text { Answer Set Programming } \\
\text { y razonamiento de sentido } \\
\text { común }\end{array}$ \\
\hline 4 & M & $\begin{array}{c}\text { Nivel } \\
\text { superior-Pública }\end{array}$ & $\begin{array}{l}\text { IV. Humanidades y } \\
\text { Ciencias de la } \\
\text { Conducta (Filosofía) }\end{array}$ & 1 & $\begin{array}{c}\text { Lógica medieval, lógica } \\
\text { modal, historia de la } \\
\text { lógica, filosofía y } \\
\text { literatura }\end{array}$ \\
\hline
\end{tabular}

Tabla 4: Características generales de los sujetos

modelar un fenómeno. Por aprioricidad, la capacidad que tiene un sistema para determinarse a sí mismo a priori. Por objetividad, que los modelos que resulten de aplicar el sistema sean objetivos con respecto a una base deductiva. Por simetría, que el sistema posea dualidad o propiedades de Galois. Y por último, por analiticidad, que el sistema sea explicable analíticamente. La aplicación del PAJ al sujeto 1 produjo el Modelo 1 (tabla 5).

\begin{tabular}{cccc}
\hline Criterios & Índice & Alternativas & Índice \\
\hline Expresividad & .5259 & Lógica tetravalente & .2593 \\
\hline Objetividad & .1962 & Lógica intuicionista & .1769 \\
\hline Simetría & .0984 & Lógica intensional & .1564 \\
\hline Aprioricidad & .0696 & Teoría de conjuntos & .1545 \\
\hline Analiticidad & .0696 & FOL & .1265 \\
\hline Explicatividad & .0404 & Lógica algebraica & .1265 \\
\hline$C R$ & & .1185 \\
\end{tabular}

Tabla 5: Modelo 1

Aunque la proporción de inconsistencia no es tan significativa $(C R>.1000)$ y tal vez sea necesario que el sujeto 1 revise algunas valoraciones, los resultados inducen un modelo de decisión en el que se prefiere, claramente y por mucho, a la expresividad sobre otras propiedades, y a los sistemas tetrava- 
lentes sobre otros porque, en última instancia, la expresividad resulta ser una propiedad más importante que otras. Inversamente, la lógica clásica (la teoría de conjuntos, FOL y la lógica algebraica) resulta ser la menos preferida, posiblemente por la misma razón, a saber, por ser menos expresiva.

Modelo 2. El sujeto 2 consideró tres sistemas y tres propiedades: FOL, PROP con una extensión modal y un sistema trivalente; y expresividad, traducibilidad y aplicabilidad. Resulta interesante que el sujeto 2 entendía por expresividad lo mismo que el sujeto 1 , si bien ambos sujetos trabajan temas diferentes en áreas diferentes: la capacidad de un sistema para representar información. Por traducibilidad se entiende la capacidad de un sistema para expresar otros sistemas en términos del primero. Y por último, por aplicabilidad, la capacidad de un sistema para ser computacionalmente implementado. La aplicación del PAJ al sujeto 2 produjo el Modelo 2 (tabla 6).

\begin{tabular}{cccc}
\hline Criterios & Índice & Alternativas & Índice \\
\hline Expresividad & .6584 & PROP modal & .6504 \\
\hline Aplicabilidad & .2344 & Lógica trivalente & .2409 \\
\hline Traducibilidad & .0802 & FOL & .1088 \\
\hline$C R$ & \multicolumn{3}{c}{} \\
\hline
\end{tabular}

Tabla 6: Modelo 2

Como en el caso anterior, aunque la proporción de inconsistencia (CR> .1000) no es tan significativa, nos indica que tal vez es necesario revisar algunas valuaciones. Dicho esto, este resultado induce un modelo en el que se prefiere a la expresividad sobre otras propiedades y en el que se prefiere a la lógica modal proposicional sobre otros sistemas porque, en última instancia, la expresividad resulta más importante que el resto de criterios. Adicionalmente, como en el caso anterior, la lógica clásica (FOL) se menciona pero resulta ser la menos preferida.

Modelo 3. El sujeto 3 consideró sólo dos sistemas, PROP y un sistema multivaluado; y tres propiedades: ejemplaridad, aplicabilidad y comprensión. Por ejemplaridad se entiende la idea de que los estudios sobre el sistema en cuestión tengan profundidad paradigmática. Por aplicabilidad, lo mismo que entendía el sujeto 2: que el sistema sea susceptible de una implementación computacional. Y por último, por comprensión, que el entendimiento de los 
mecanismos inferenciales del sistema permitan la comprensión de los mecanismos de otros sistemas. La aplicación del PAJ al sujeto 3 produjo el Modelo 3 (tabla 7).

\begin{tabular}{cccc}
\hline Criterios & Índice & Alternativas & Índice \\
\hline Ejemplaridad & .6798 & Lógica multivaluada & .6199 \\
\hline Comprensión & .2203 & PROP/FOL & .3801 \\
\hline Aplicabilidad & .0999 & & \\
\hline$C R$ & & .6821 & \\
\hline
\end{tabular}

Tabla 7: Modelo 3

En este caso la proporción de inconsistencia $(C R>.1000)$ nos indica que es necesario revisar las valoraciones, sin embargo, los resultados inducen un modelo de decisión en el que se prefiere a la ejemplaridad sobre otras propiedades y en el que se prefiere a la lógica multivaluada sobre otros sistemas. No es claro $(C R=.6821)$, sin embargo, por qué es esta la elección adecuada si PROP es más ejemplar que la lógica multivaluada.

Modelo 4. El sujeto 4 consideró tres familias o clases de lógicas, más que sistemas: la lógica clásica (FOL), la lógica no-clásica y la lógica informal. Además consideró cuatro propiedades: comprensión, extensibilidad, naturalidad y axiomatización. Por comprensión entiendía lo mismo que el sujeto anterior, a saber, que el entendimiento de los mecanismos inferenciales del sistema permitan la comprensión de los mecanismos de otros sistemas. Por extensibilidad, que el sistema soporte extensiones. Por naturalidad, que la teoría de prueba del sistema sea expresable mediante reglas de deducción natural. Y por último, por axiomatización, que el sistema se pueda definir axiomáticamente. La aplicación del PAJ al sujeto 4 produjo el Modelo 4 (tabla 8).

A diferencia de los casos anteriores, este modelo muestra consistencia interna $(C R<.1000)$ y los resultados inducen un modelo de decisión en el que se prefiere, por mucho, a la comprensión sobre otras propiedades y que, por esta razón, se prefiere a la lógica clásica sobre otros sistemas y, al contrario, por el ordenamiento de las propiedades, la lógica informal resulta ser la elección menos preferida.

\section{Conclusiones}

A manera de conclusión, consideremos cinco comentarios finales. 


\begin{tabular}{cccc}
\hline Criterios & Índice & Alternativas & Índice \\
\hline Comprensión & .6574 & Lógica clásica & .5318 \\
\hline Axiomatización & .2027 & Lógicas no-clásica & .3462 \\
\hline Naturalidad & .0942 & Lógica informal & .1219 \\
\hline Extensibilidad & .0457 & & \\
\hline$C R$ & & .0740 \\
\hline
\end{tabular}

Tabla 8: Modelo 4

a. La alternativa invariable. Aun en un contexto de pluralidad, parece que la alternativa más considerada sigue siendo la lógica clásica, con cuatro menciones de cuatro. Lo relevante de esta elección, sin embargo, no es tanto el número de menciones sino el hecho de que todas y todos consideran a la lógica clásica como una alternativa bona fide, aunque no siempre sea la ganadora.

Esto es de suyo interesante pero también parece ofrecer evidencia empírica a favor de la hipótesis que afirma que la lógica clásica es invariable con respecto al área de estudio o los intereses de investigación (cf. Williamson, 2017); no obstante, una conclusión como la anterior sería espuria porque del hecho de que todas y todos, invariablemente, hayan tomado en cuenta a la lógica clásica no se sigue que la invariabilidad sea una razón suficiente para considerarla como la alternativa ganadora, ya no digamos la alternativa invariable, al menos por dos razones: $i$ ) los motivos detrás de la elección de la lógica clásica parecen obedecer más a un estándar de la didáctica tradicional de la lógica en las instituciones de educación superior mexicanas que a la práctica diaria de las y los investigadores, como veremos en los siguientes comentarios; y ii) si bien las lógicas no-clásicas que mencionan las y los investigadores no se pueden agrupar a través de la etiqueta de un único sistema no-clásico, como decir, por ejemplo, "lógica tetravalente", todas y todos nombran, además de la lógica clásica, por lo menos una lógica no-clásica también, por lo que sería impreciso concluir que la lógica clásica es la alternativa invariable sólo porque no se menciona una sola lógica no-clásica de manera uniforme o constante (volveremos a mencionar esto más adelante).

b. Los criterios ganadores. Habiendo hecho esta última aclaración, existe, sin embargo, una compensación interesante para el caso de la lógica clásica: ciertamente, es el sistema más mencionado de manera unívoca pero es el que menos gana. Una posible explicación de esta situación tiene que ver con 
la elección de criterios o propiedades. Los criterios más considerados por las y los investigadores son la comprensión (dos menciones de cuatro), la expresividad (dos menciones de cuatro) y la aplicabilidad (dos menciones de cuatro).

Esta elección de propiedades puede ayudarnos a explicar la situación del comentario anterior: aunque la lógica clásica parece ser la alternativa invariable por la comprensión referencial que facilita al estudiar otros sistemas, no resulta ser la alternativa ganadora global porque las y los lógicos valoran, además de la comprensión, otras propiedades relevantes para la resolución de problemas, como la expresividad y la aplicabilidad, y en ciertos contextos lingüísticos y computacionales, otros sistemas no-clásicos resultan ser más expresivos o aplicables que la lógica clásica.

Esto sugiere que la elección de sistemas depende de la valoración de ciertas propiedades y que la elección de tales propiedades depende, en buena medida, no sólo de las cualidades propias de los sistemas en cuestión sino de las prácticas de las y los lógicos.

c. Las propiedades en la práctica. En efecto, los modelos de decisión inducidos a través de las aplicaciones del PAJ muestran ciertas variaciones sobre las propiedades metalógicas. Parece que poco importa si los sistemas en cuestión tienen las propiedades metalógicas usuales (como las que se mencionaron en la sección 3), posiblemente porque se asume que los sistemas en cuestión ya tienen algunas de estas; antes bien, lo que resulta apremiante es que las propiedades de interés que las y los lógicos valoran son propiedades como comprensión, expresividad, traducibilidad y aplicabilidad.

Ahora bien, además de que podemos indicar con claridad cuáles de estas propiedades resultan ganadoras, nos parece más interesante notar la distribución de propiedades que se asume en la práctica diaria de la lógica, pues esta distribución tiene que ver más con las preocupaciones e intereses propios de la docencia y la investigación de cada disciplina y cada área que con una característica intrínseca de cada sistema. Ciertamente, si las prácticas propias de cada disciplina o área fueran irrelevantes para la elección de sistemas lógicos esperaríamos ver una distribución de propiedades más o menos uniforme con respecto a las áreas de investigación; sin embargo, los resultados muestran que sólo dos propiedades de doce son compartidas (comprensión y expresividad), mientras que el resto, diez de doce, es disjunto (tabla 9). 


\begin{tabular}{cccc}
\hline Propiedad & Área VII & Área IV & Total \\
\hline Comprensión & 1 & 1 & 2 \\
\hline Expresividad & 1 & 1 & 2 \\
\hline Aplicabilidad & 2 & - & 2 \\
\hline Ejemplaridad & 1 & - & 1 \\
\hline Traducibilidad & 1 & - & 1 \\
\hline Axiomatización & - & 1 & 1 \\
\hline Naturalidad & - & 1 & 1 \\
\hline Objetividad & - & 1 & 1 \\
\hline Simetría & - & 1 & 1 \\
\hline Aprioricidad & - & 1 & 1 \\
\hline Analiticidad & - & 1 & 1 \\
\hline Explicatividad & - & 1 & 1 \\
\hline Total & 6 & 9 & 15 \\
\hline
\end{tabular}

Tabla 9: Distribución de propiedades

d. Las alternativas en la práctica. De manera similar, los modelos de decisión inducidos a través de las aplicaciones del PAJ muestran que, en la práctica, aunque la lógica clásica es básica, no resulta ser la mejor valorada. Las menciones de sistemas no-clásicos (seis) o informales (seis más uno) superan a las menciones de sistemas clásicos (cuatro), independientemente del área (tabla 10).

\begin{tabular}{cccc}
\hline Sistema & Área VII & Área IV & Total \\
\hline Multivaluado & 2 & 1 & 3 \\
\hline Intensional & 1 & 1 & 2 \\
\hline Intuicionista & - & 1 & 1 \\
\hline Clásico & 2 & 2 & 4 \\
\hline Informal & - & 1 & 1 \\
\hline Total & 5 & 6 & 11 \\
\hline
\end{tabular}

Tabla 10: Distribución de sistemas (I)

Más aún, si hacemos una partición menos granular (excluyendo a la lógica informal, que parece ser más una familia de teorías que un sistema) obtenemos 
la distribución de la tabla 11, la cual sugiere que, aún entre distintas áreas o disciplinas, el uso de sistemas clásicos y no-clásicos es indistinto, como mencionábamos en el primer comentario, si bien la consideración de propiedades tiende a ser más bien disjunta. Así pues, este resultado y el anterior sugieren que, aunque la distribución de sistemas es más bien uniforme (y por ende la lógica clásica es tan invariable como las lógicas no-clásicas), la distribución de propiedades no lo es (puesto que esta distribución depende de las prácticas de las y los lógicos).

\begin{tabular}{cccc}
\hline Sistema & Área VII & Área IV & Total \\
\hline Clásico & 2 & 3 & 5 \\
\hline No-clásico & 3 & 2 & 5 \\
\hline Total & 5 & 5 & 10 \\
\hline \multicolumn{4}{c}{ Tabla 11: Distribución de sistemas (II) }
\end{tabular}

e. El pluralismo lógico revisitado. A partir de una interpretación de estos resultados podemos ofrecer una breve reflexión sobre el pluralismo lógico. Comencemos por hacer eco de una expresión post-kuhninana: si ciencia es lo que hacen las y los científicos, supongamos que la lógica es también lo que hacen las y los lógicos. Si esto es así, entonces prestar atención a la práctica diaria de la lógica en la docencia y la investigación puede ofrecer evidencias o argumentos para el debate sobre el pluralismo lógico.

Para explorar la práctica diaria de la lógica podemos preguntarnos qué hacen las y los lógicos cuando hacen lógica y qué es lo que les resulta importante en su práctica diaria. Según nuestros resultados, podemos responder la primera pregunta afirmando que las y los lógicos resuelven problemas propios de sus disciplinas; y podemos responder la segunda pregunta diciendo que lo que importa en la práctica son las propiedades metalógicas que ayudan a responder problemas disciplinares.

En efecto, nuestros resultados inducen modelos de decisión que muestran que las y los lógicos, en su práctica diaria, valoran ciertas propiedades metalógicas sobre otras, y si bien algunas de estas propiedades se comparten entre áreas y disciplinas, la distribución de propiedades no es uniforme y parece obedecer a las prácticas propias de cada una de ellas: el interés por la 
traducibilidad y la aplicabilidad es propio del área VII, por ejemplo, mientras que el interés por la simetría y la axiomatización es propio del área IV.

Esto ofrece evidencia de que la práctica diaria de la lógica favorece una interpretación pluralista de la misma frente a visiones más monistas de la lógica (además de que vulnera el argumento típico de que la lógica clásica es invariable a la disciplina, como notamos en el comentario $a$ ); sin embargo, a diferencia de otras versiones de pluralismo lógico como el basado en casos (Beall y Restall, 2006), el basado en constantes (Varzi, 2002) o el basado en normas epistémicas (Field, 2009), el tipo de pluralismo que nuestros modelos sugieren es un pluralismo basado en las propiedades metalógicas que emergen de la práctica diaria de la lógica.

Esta forma de pluralismo bien podría llamarse pluralismo pragmático para distinguirlo de un instrumentalismo o de un relativismo lógico porque, si bien la práctica de la lógica depende de ciertas metas (como argumentaría un instrumentalismo) propias de cada disciplina (como argumentaría un relativismo), la distribución de propiedades metalógicas sugiere que, en última instancia, aunque la práctica de la lógica sí es variable con respecto a metas y disciplinas, tiene límites en términos, por ejemplo, de expresividad y comprensión. En otras palabras, bajo esta forma de pluralismo no diríamos, por ejemplo, que hay más de una lógica, más bien diríamos que hay más de una propiedad metalógica de interés. En este caso, nuestros resultados sugieren que la expresividad y la comprensión son límites ideales de la investigación en lógica para obtener sistemas lógicos: que haya más de una lógica es consecuencia de estos límites y no al revés.

Por supuesto, como esta forma de pluralismo ha sido generada inductivamente a partir de una pequeña muestra de lógicas y lógicos de México, no descartamos que una muestra más grande y representativa pueda arrojar otros resultados; sin embargo, creemos que esto no afectaría la idea de un pluralismo pragmático en lógica: al contrario, nos ayudaría a definir de manera más fina algunos otros límites de la investigación en lógica.

\section{Referencias}

Beall, J.C. y Restall, G., (1996), Logical Pluralism,Oxford University Press, Oxford. —, (2000), "Logical Pluralism", Australasian Journal of Philosophy 78, 475-493.

Eklund, M., (1996), “On how logic became first order”, Nordic Journal of Philosophical Logic, 1 (2), 147-167.

Field, H., (2009), "Pluralism in logic", The Review of Symbolic Logic, 2(2). 
Goddu, G.C., (2002), "What is exactly logical pluralism?", Australasian Journal of Philosophy, 80 (2), 218-230.

Haack, S., (1978), Philosophy of Logics, Cambridge University Press, Cambridge.

Hughes, G. E., y Cresswell, M. J., (1996), A New Introduction to Modal Logic, Psychology Press, Londres.

Moss, L. "Natural Logic", en: S. Lappin y C. Fox (Eds.), The Handbook of Contemporary Semantic Theory (pp. 561-591, Ch. 18), John Wiley \& Sons.

Saaty, Th, (2008), "Relative Measurement and its Generalization in Decision Making. Why Pairwise Comparisons are Central in Mathematics for the Measurement of Intangible Factors. The Analytic Hierarchy/Network Process", Rev. R. Acad. Cien, 102 (2), 251-318.

Varzi, A.C., (2002), “On logical relativity”, Philosophical Issues, 12, 197-219.

Williamson, T., (2017), "Semantic Paradoxes and Abductive Methodology”, en: B. D. Amour-Garb (Ed.), Reflections on the Liar, Oxford University Press, Oxford. 